Published in final edited form as:

Intellect Dev Disabil. 2012 February ; 50(1): 2-15. doi:10.1352/1934-9556-50.1.2.

\title{
Timing of first dental recall visits for newly Medicaid-enrolled children with an intellectual or developmental disability in lowa
}

\author{
Donald L. Chi, DDS ${ }^{\star}$, Elizabeth T. Momany, PhD ${ }^{\star}$, Michael P. Jones, PhD\&, Raymond A. \\ Kuthy, DDS, MPH ${ }^{*}, \#$, and Peter C. Damiano, DDS, MPH $^{*}, \#$ \\ "Public Policy Center, The University of lowa \\ 'College of Dentistry, Department of Pediatric Dentistry, The University of lowa \\ \#College of Dentistry, Department of Preventive and Community Dentistry, The University of lowa \\ ${ }^{\&}$ College of Public Health, Department of Biostatistics, The University of lowa
}

\begin{abstract}
This study compared the extent to which having an intellectual or developmental disability (IDD) is associated with the rates at which newly Iowa Medicaid-enrolled children ages 3-8 had a first dental recall visit. We used survival analytic techniques to test our hypothesis that children with an IDD would have later first dental recall visits than children without an IDD. Our results suggest no significant difference in the time to first dental recall for children by IDD status ( $p=.99)$. After adjusting for covariates, the only factor associated with earlier first dental recalls was the length of time from enrollment in Medicaid to the first comprehensive dental visit. Children for whom it took $>13$ months to see a dentist for their first comprehensive dental visit were 1.68 times as likely to have an earlier first dental recall as children who had their first comprehensive dental visit within 4 months of enrolling in Medicaid ( $p<.0001)$. We found that having an IDD was not associated with later first dental recall visits for newly Medicaid-enrolled children ages 3-8 in Iowa. Future work should identify other factors associated with poor oral health for Medicaidenrolled children with an IDD.
\end{abstract}

\section{Keywords}

Medicaid; children with special health care needs; access to care; dental utilization; survival analysis

\section{Introduction}

As highlighted in the Institute of Medicine's 2001 publication, Crossing the Quality Chasm, the timely receipt of health care services is critical in disease prevention (Institute of Medicine 2001). While dental visits were not explicitly mentioned in this report, the timing of dental recall visits (e.g., checkups) is important in preventing oral health problems in children, especially for those at increased risk for oral health problems. Timely dental recall visits (DRVs) enable high-risk children to benefit from anticipatory guidance (Plutzer \& Spencer, 2008; Zanin, Menehim, Assaf, Cortellazzi, \& Pereira, 2007; Davies, Duxbury, Boothman, Davies, \& Blinkhorn, 2005; Nainar \& Mohummed, 2004) and preventive care such as pit and fissure sealants and topical fluoride (Ahovuo-Saloranta, Hiiri, Borblad, Mäkelä, \& Worthington, 2008; Marinho, Higgin, Logan, \& Sheiham, 2003; Weintraub, Ramos-Gomez, Jue, Shain, Hoover, Featherstone, \& Gansky, 2006). DRVs also help to reduce the physical and social morbidities associated with untreated tooth decay such as pain, local and systemic infection, school absences, and low self-esteem (Vargas, Macek, 
Goodman, \& Wagner, 2005; Gift, Reisine, \& Larach, 1992; Cunnion, Spiro, Jones, Risk, Papageorgious, Tate, Casamassimo, Hayes, \& Garcia, 2010).

Children identified with an intellectual or developmental disability (IDD) are at increased risk for poor oral health. For example, some take prescription medications that contain high concentrations of sugar or lead to chronic xerostomia - factors associated with dental caries (Feigal, Jensen, \& Mensing, 1981; Keene, Galasko, \& Land, 2003). Many have highly cariogenic diets (Nunn, Braunstein, Krall Kaye, Dietrich, Garcia, \& Henshaw, 2009; Marshall, Eichenberger-Gilmore, Larson, Warren, \& Levy, 2007). Others have behavioral co-morbidities that make it difficult for caregivers to brush the child's teeth and gums regularly with fluoridated toothpaste (Ferguson \& Cinotti, 2009). On a provider-level, some dentists are unwilling to see children with an IDD because of inadequate training (Casamassimo, Seale, \& Ruehs, 2004). In addition, many children with an IDD are enrolled in state Medicaid programs, introducing an additional set of system-level barriers that make access to timely DRVs difficult (Nainar, 2000; Mayer. Stearns, Norton, \& Rozier, 2000; Al Agili, Pass, Bronstein, \& Lockwood, 2007).

Children with an IDD who have delayed DRVs may be at increased risk for dental caries (Maurer, Boggs, Mourino, \& Farrington, 1996). Another publication reported no difference in the likelihood of preventive dental care utilization for Iowa Medicaid-enrolled children ages 3-17 by IDD status (Chi, Momany, Kuthy, Chalmers, \& Damiano, 2010a). One limitation of this study was the failure to adjust for overall health status. In a more recent study, which addressed this limitation, researchers found that newly Medicaid-enrolled children ages 3-8 with an IDD in Iowa were significantly more likely to have later first dental visits than those without an IDD ( $p=.04)$ (Chi, Momany, Jones, \& Damiano, 2010b).

Even though timely DRVs are important in preventing oral health problems, no studies to date have compared the rates at which the first DRV take place after the first dental visit for Medicaid-enrolled children by IDD status. Without knowledge on the timing of first DRVs for Medicaid-enrolled children, it is not possible to develop evidence-based clinical interventions and policies aimed at improving access to appropriate dental care for this vulnerable population.

The primary objective of this study was to compare the rates at which Medicaid-enrolled children with and without an IDD had the first DRV after the first comprehensive dental visit. In addition, we sought to identify the factors associated with the rates at which Medicaid-enrolled children had the first DRV. Based on previous work that Medicaidenrolled children with an IDD had significantly later first dental visits (Chi et al., 2010b), we hypothesized that Medicaid-enrolled children with an IDD would have later first DRVs than children without an IDD. Furthermore, we hypothesized that other factors from our conceptual model would also be important determinants of the time to first DRVs for all Medicaid-enrolled children.

\section{Methods}

\section{Conceptual Model}

We adapted a sociocultural oral health disparities model proposed by Patrick et al. (2006) as the conceptual model for this study. We evaluated factors from the following domains as potential determinants of the time to first DRV:

- $\quad$ ascribed (IDD status, age, sex, overall health status);

- proximal (use of restorative dental care, use of preventive medical care, number of months from enrollment in Medicaid to the first comprehensive dental visit) 
- immediate (whether the child had a Medicaid-enrolled adult and/or sibling);

- intermediate (rurality);

- distal (whether the child lived in a dental Health Professional Shortage Area).

\section{Study Design}

This was a retrospective cohort study based on administrative data. This study was approved by the Institutional Review Board of the University of Iowa.

\section{Data Source}

We analyzed Iowa Medicaid enrollment and claims data from calendar years 2005-2007. The child was the unit of analysis. The enrollment files contained the child's unique identification number (used to link the files), age, sex, county of residence, and zip code at baseline. "Baseline" was defined as the child's first month of enrollment in the Iowa Medicaid program in 2005. Enrollment and medical claims files were used to identify IDD status. The dental claims files contained information on all dental services received by the child and for which a claim was submitted by a dentist.

\section{Sample}

We focused on children ages 3-8 who were newly enrolled in the Iowa Medicaid program in calendar year 2005. A child was classified as "newly enrolled" if he or she had no evidence of enrollment in the Iowa Medicaid program in the 12 months prior to the first month of Medicaid enrollment in 2005. We restricted our study to children ages 3-8 because of the teeth expected to be present clinically (primary dentition or early mixed dentition consisting of primary teeth, permanent incisors, and first molars). We excluded children living in institutions to focus on those seeking dental care from community-based dentists. Children enrolled in Medicaid through the foster care program were excluded because these enrollees are known to have heterogeneous dental utilization patterns (Colthirst, Momany, Damiano, $\&$ Warren, 2009). In addition, we excluded children under age 3 years because IDD is typically identified after the third birthday (Council on Children with Disabilities, 2006; Pinto-Martin, Dunkel, Earls, Fliedner, \& Landes, 2005). Our final study population consisted of children who received a comprehensive dental examination after enrolling in the Iowa Medicaid program with no other dental visits prior to this first comprehensive dental examination $(\mathrm{N}=2,982)$.

\section{Independent Variable}

IDD status (no/yes) was the main independent variable. We used four of the five criteria described previously by Chi et al. (2010a, 2010b) to operationalize IDD. These criteria were developed by a group of physicians, dentists, and developmental psychologists with extensive clinical experience treating children with special needs at the University of Iowa Center for Disability and Development and program staff members at the Iowa Medicaid administrative office. We used both a categorical (International Classification of Disease, Ninth Revision, Clinical Modification [ICD-9-CM] medical diagnostic codes indicating a non-acquired cognitive deficit) and a non-categorical approach (participation in the State Home and Community Based Waiver Program for the Mentally Retarded, recipients of Targeted Case Management services for the mentally retarded, and children with evidence of an IDD exception indicator as noted by a physician). These data were found in the child's eligibility and medical claims files from calendar year 2005. 


\section{Dependent Variable}

The dependent variable was the time to first DRV after the first comprehensive dental examination (Figure 1). Five-digit alphanumeric Current Dental Terminology (CDT) Codes were used to identify specific dental services from each child's dental claims files. In this study, the presence of a valid CDT Code was equivalent to a dental visit.

A child was considered to have had a comprehensive dental visit if CDT Codes D0150 or D0110 appeared in the child's dental claims files after initially enrolling into the Iowa Medicaid program. There must have been no evidence of any other previous dental care received prior to this first comprehensive dental visit. The first DRV was defined by CDT Codes D0120 or D0150, which had to appear anytime after the first comprehensive dental visit. To account for the effect of provider switching, the two dental visits must have been filed by the same dentist.

\section{Model Covariates}

We included three ascribed factors: age at baseline (continuous variable), sex (female/male), and overall health status (healthy/any acute condition but no chronic condition/any chronic condition). Overall health status was defined using the 3M Clinical Risk Grouping (CRG) methodology (Hughes, Averill, Eisenhandler, Goldfield, Muldoon, Neff, \& Gay, 2004). The CRG uses medical claims data and classifies children into one of the following mutuallyexclusive, hierarchical core health status groups (CHSGs): healthy, acute condition, single minor chronic condition (CC), minor CC in multiple systems, single dominant or moderate $\mathrm{CC}$, significant $\mathrm{CC}$ in multiple systems, dominant $\mathrm{CC}$ in three or more systems, malignancy, and catastrophic CC. Children from the first CHSG were classified as healthy, those from the second CHSG were classified as having any acute condition but no CC, and the remaining children were classified as having any $\mathrm{CC}$.

We also included three dichotomous proximal factors: whether the child received any restorative dental care between the first comprehensive dental visit and the first DRV, whether the child utilized preventive medical care in 2005, and the number of months elapsed from enrollment in Medicaid to the first comprehensive dental visit ( $<4$ months, 4-6 months, 7-12 months, $>12$ months). Children who received any restorative care (defined by CDT Codes D1510-D1550; D2110-D2394; D2930-D4342; D7110-D7140; D9420) on the same date as the first DRV were classified as not having received restorative dental care, unless restorative dental care was received after the date of the first DRV. This was to ensure that the first DRV was initiated as a preventive visit rather than because of a dental problem (e.g., toothache, broken filling, abscess).

In addition, we included two dichotomous immediate factors (whether the child lived in a household with a Medicaid-enrolled adult and whether there was another Medicaid-enrolled sibling in the household); one intermediate factor (rurality of the child's county of residence at baseline as a four-level categorical variable based on the 2003 U.S. Department of Agriculture Rural-Urban Continuum Codes: metropolitan, urban adjacent to metropolitan, urban not adjacent to metropolitan, rural); and one dichotomous distal factor (whether the child lived in a dental Health Professional Shortage Area [HPSA] at baseline,.

\section{Analysis}

The chi-square test and t-test was used to assess for statistical differences in proportions and means, respectively. Kaplan-Meier survival curves were constructed and the log-rank test was used to identify the relationship between IDD status and the time to first DRV ( $a=0.05$ ), two approaches that adjust for censoring but not for other model covariates. We assessed for collinearity between covariates measuring similar domains (e.g., rurality and dental HPSA). 
There was no evidence of collinearity. We tested the proportional hazards assumption for each model covariate with time-dependent variables. No variables violated the proportional hazards assumption. We also tested for an interaction between the two immediate variables (having a Medicaid-enrolled sibling and/or adult from the household). Because this interaction failed to reach statistical significance ( $p=.71$ ), it was excluded from the final regression models. Multiple variable Cox proportional hazards regression models were generated $(a=0.05)$. Two additional regression models were constructed to adjust for potential correlation between subjects from the same household and children seen by the same dentist. However, because this adjustment did not affect the hazard ratios, we only reported hazards ratios from the unadjusted model. All data were analyzed using SAS 9.2 for Windows (PROC LIFETEST and PROC PHREG statements).

\section{Results}

Descriptive Data. About 1.3\% of newly Iowa Medicaid-enrolled children ages 3-8 were identified with an IDD (Table 1). On average, children with an IDD were enrolled in Medicaid five months longer than those without an IDD (29.7 months and 24.6 months, respectively) though this difference was not significant $(p=.455)$. Significantly larger proportions of children without IDD had another Medicaid-enrolled sibling $(81.6 \%)$ or adult $(60.2 \%)$ in the household than those with IDD (48.7\% and 30.8\%, respectively).

\section{Dental Utilization}

About $53 \%$ of children without IDD and $43.6 \%$ of children with IDD had their first comprehensive dental visit within six months of enrollment in the Medicaid program (Table 1). Overall, $14.7 \%$ of newly Medicaid-enrolled children had at least one restorative dental visit between the first comprehensive dental visit and first DRV. Less than $8 \%$ of children with IDD received restorative dental care.

Table 2 displays the cumulative proportions of children with a first DRV at specified time intervals unadjusted for model covariates but adjusted for censoring. About $42 \%$ of all children had a first DRV within six months of the first comprehensive dental visit. This increased to $77 \%$ by year one, with nearly all children having a DRV within 31 months of the first comprehensive dental visit. Among children with an IDD, 40\% and 73\% had a DRV within six months and one year of the first comprehensive dental visit, respectively. These rates were slightly higher for children without an IDD (42\% and 77\%). However, over the entire study period, the difference in the time to first DRV for children with and without IDD failed to reach statistical significance $(p=.87)$ as depicted in the Kaplan-Meier survival curves (Figure 2).

\section{Hazard Models}

After adjusting for model covariates (Table 3), there was no significant difference in the time to first DRV by IDD status ( $p=.988$ ). The only factor that appeared to be statistically significant was length of time from enrollment in Medicaid to the first comprehensive dental visit. Compared to children whose first comprehensive dental visit took place within four months of enrollment, those with a first comprehensive dental visit $\geq 13$ months after enrollment were 1.68 times as likely to have a earlier first DRV $(P<.0001)$. In other words, children who took the longest to see a dentist for the first time were significantly more likely to have a first DRV sooner than those who got in quickly for their first dental visit. One immediate factor, having a Medicaid-enrolled adult in the household, approached statistical significance $(p=.064)$. 


\section{Discussion}

This is the first published study comparing the time to first DRV for newly Medicaidenrolled children with and without an intellectual or developmental disability (IDD). Timely DRVs are important, especially for children at risk for oral health problems, because dentists can intervene with regular preventive care and less invasive restorative treatments when necessary. During DRVs, caregivers of these children are supposed to receive anticipatory guidance, which reinforces positive oral health care practices that can benefit their children. We hypothesized that newly Medicaid-enrolled children with an IDD would have later first DRVs than children without IDD. However, our regression model results revealed no significant difference in the time to first DRV by IDD status. The only factor that was significantly related to earlier first DRVs was the time to the first comprehensive dental visit after enrolling into the Medicaid program. Children with a first comprehensive dental visit $>12$ months after enrolling into Medicaid were 1.68 times as likely to have an earlier first DRV as children with a first comprehensive dental visit $<4$ months of enrollment.

While not directly comparable, a previous study on dental recall rates reported that $64 \%$ of patients ages 2-46 (mean age 14.2 years) with a handicap such as mental retardation, cerebral palsy, epilepsy, or an orthopedic problem had a recall within 12 months (Maurer et al., 1996). This is about $13 \%$ less than the proportion of children in our study with a dental recall within 12 months (77\%). In addition, 55.3\% of newly Medicaid-enrolled children ages $3-8$ in our study had a comprehensive dental visit followed by a RDV $(2,982 / 5,391)$ whereas $12.6 \%$ of Medicaid-enrolled children <21 years in New Hampshire received two dental examinations (Chi \& Milgrom, 2009). There are no other published studies in the literature to which we can compare our findings on first DRV rates for newly Medicaidenrolled children by IDD status.

We have three potential explanations of our failure to detect a difference in the time to first DRVs for Medicaid-enrolled children by IDD status. First, it may be that once a newly Medicaid-enrolled child sees a dentist for the first time and is not referred to another dentist, two traits shared by children in this study, IDD status is a less important determinant of the timing of subsequent DRVs. Second, $41 \%$ of children identified with an IDD were classified as healthy, a relatively large subgroup of children who may be similar to those without IDD. Dental utilization for these children could have skewed the outcome measure for children with an IDD toward unity. However, we reanalyzed our data after excluding these healthy children with an IDD and found that the adjusted hazard ratios remained the same, making this explanation unlikely. Third, while the difference was not statistically significant, 7.7\% of children with an IDD received restorative treatment between the first comprehensive dental visit and the first DRV compared to $14.8 \%$ of children without IDD (Table 1). This suggests that children with an IDD in our study had less need for restorative care, in which case we would expect to similar rates of first DRVs by IDD status.

\section{Other Determinants of the Time to First DRVs for All Children}

\section{Ascribed Factors}

In addition to IDD status, we examined three ascribed factors: age, sex, and overall health status. We failed to detect a significant difference by age in the time to first DRV. This is inconsistent with previous findings that younger Medicaid-enrolled children are significantly more likely to have later first dental visits than those who are older (Chi et al., 2010b).

Disregarding statistical significance, the directions of the hazard ratios reveal that children ages 3, 4 and 5 were less likely to have earlier first DRVs than 8-year-olds. We also found no differences by sex or overall health status. While there is no reason to expect heterogeneous dental utilization by sex, it is unclear why children with a chronic condition 
were no less likely to have earlier first DRVs than healthy children, given that children in the former group have other health care needs that may be higher priority than oral health. The relationship between overall health status and dental utilization for Medicaid-enrolled children warrants further investigation.

\section{Proximal Factors}

There were three proximal factors included in our models: use of primary medical care, time elapsed from enrollment in Medicaid to the first comprehensive dental visit, and whether the child received any restorative dental care from the first comprehensive dental visit to the first DRV. The only factor that was significant was the time elapsed from enrollment to the first comprehensive dental visit. Even then, only children who had a first comprehensive dental visit $>12$ months from the time of enrollment were significantly more likely to have earlier first DRVs than children with a first comprehensive dental visit $<4$ months from enrollment. While it is possible that this difference was due to chance (type I error), another explanation is that the presence of risk factors for dental caries such as inflamed gums and white spot lesions (reversible cavities) might prompt dentists to assign these children to more frequent DRVs. In addition, caregivers who postpone the child's first visit may learn about the importance of periodic DRVs and subsequently be more vigilant about coming in for timely DRVs. As for the other two proximal factors, we believe the explanation for a lack of statistical significance is that the first dental comprehensive visit may be the single most important determinant of the timing of the first DRV.

\section{Immediate Factors}

We examined two immediate factors: whether the child had a Medicaid-enrolled sibling or a Medicaid-enrolled adult in the household. Both variables as well as the interaction between the two variables failed to reach statistical significance. However, the adult variable approached significance ( $p=.06$ ) suggesting that children with a Medicaid-enrolled adult may be less likely to have earlier first DRVs. Households with a Medicaid-enrolled adult are among the lowest income families in Medicaid. This economic vulnerability may be associated with caregiver health beliefs and behaviors that lead to poor dental utilization. As a result, children from these households may experience additional barriers to timely dental recalls such as high caregiver stress, unreliable transportation, and inability of the primary caregiver to take time off from work. Given the link between caregiver and child dental utilization (Grembowski, Spiekerman, \& Milgrom, 2008) and the importance of monitoring the oral health of vulnerable children, the relationship between immediate factors and the timing of first DRVs requires further clarification.

\section{Intermediate Factor}

We examined the role of one intermediate factor, rurality of the child's county of residence, and failed to detect statistically significant differences across the four rurality groups. This conflicts with previous findings suggesting that children living in rural areas have problems accessing dental care (Devoe, Krois, \& Stenger, 2009). An explanation for our finding is that rurality may be a less important determinant of the timing of first DRVs once a child is able to establish a dental home by having a first comprehensive dental visit.

\section{Distal Factor}

There was one distal factor in our models: whether the child lived in a dental HPSA. The explanation for the lack of statistical significance is similar to the explanation for our intermediate factor (rurality). 


\section{Overall Conceptual Model}

It appears that the adapted Patrick et al. model on oral health disparities was not sufficient in helping us to identify the determinants of first DRVs for Medicaid-enrolled children. The more important finding, in the context of previous findings, is that the determinants of utilization for different types of dental care (first comprehensive dental visits and first DRVs) are heterogeneous. This underscores the complexity of modeling access for vulnerable children. Additional conceptual work may be necessary to link access to different types of dental care into a single theoretical framework, which can then be used to develop policies and clinical interventions aimed at improving access to appropriate types of dental care for all Medicaid-enrolled children.

There are three potential limitations with the current study. First, our findings can only be generalized to newly Medicaid-enrolled children ages 3-8 years. It is possible that the relationship between IDD status and the time to first DRV is different for older Medicaidenrolled children. Second, we were unable to include in our regression models behavioral measures such as caregiver attitudes, knowledge, and beliefs regarding DRVs, which are presumed to be important determinants of disparities in access to dental care in Patrick et al.'s model on oral health disparities. Future studies should collect primary data from caregivers of Medicaid-enrolled children and assess the role behavioral factors have on the time to first DRVs. Third, the lack of clinical data for children precluded an assessment of the level of dental treatment need for children in the study. We included a restorative treatment variable in our model and assumed that children who needed dental treatment received it, though this could not be verified. For this reason, future studies should collect clinical data in order to assess the extent to which the DRVs are associated with lower levels of unmet dental need subsequently. It may be that children at risk for oral health problems, such as Medicaid enrollees with an IDD, may need to see a dentist more frequently to ensure that an adequate level of preventive care is provided and to reinforce healthy oral healthrelated habits to caregivers.

In conclusion, our findings suggest no difference in the time to first DRVs for newly Medicaid-enrolled children with and without IDD. The only factor that was associated with earlier first DRVs was children for whom it took the longest (>12 months) to have their first comprehensive dental visit after enrollment into the Medicaid program. While it is encouraging that most children in our study eventually had a first DRV, over one-fifth of children had the first DRV greater than one year after the first comprehensive dental visit. If the longer periodicity of DRVs was based on lower risk for developing caries and other oral health problem, this might reflect appropriate use of dental care services. However, it is more likely that many of these children have delayed first DRVs, highlighting the larger problem that subgroups of Medicaid-enrolled children have in terms of having timely DRVs. Future work should focus on constructing additional conceptual models on access to dental care that can be used to identify the determinants of DRVs, especially for children at increased risk for oral health problems. This knowledge can then be used to develop clinical interventions and periodicity policies aimed at improving the oral health for the most vulnerable child Medicaid enrollees.

\section{References}

Ahovuo-Saloranta A, Hiiri A, Nordblad A, Mäkelä M, Worthington HV. Pit and fissure sealants for preventing dental decay in the permanent teeth of children and adolescents. Cochrane Database Systematic Review. 2008; 8:CD001830.

Al Agili DE, Pass MA, Bronstein JM, Lockwood SA. Medicaid participation by private dentists in Alabama. Pediatric Dentistry. 2007; 29:293-302. [PubMed: 17867394] 
Casamassimo P, Seale NS, Ruehs K. General dentists' perceptions of educational and treatment issues affecting access to care for children with special health care needs. Journal of Dental Education. 2004; 68:23-8. [PubMed: 14761169]

Chi DL, Momany ET, Kuthy RA, Chalmers JM, Damiano PC. Preventive dental utilization for Medicaid-enrolled children in Iowa identified with intellectual and/or developmental disability. Journal of Public Health Dentistry. 2010a; 70:35-44. Epub 2009 Aug 20. [PubMed: 19694935]

Chi D, Momany E, Jones M, Damiano P. Timing of first dental visits for newly Medicaid-enrolled children with an intellectual or developmental disability in Iowa, 2005-2007. American Journal of Public Health. 2010b In press.

Chi D, Milgrom P. Preventive dental service utilization for Medicaid-enrolled children in New Hampshire: a comparison of care provided by pediatric dentists and general dentists. Journal of Health Care for the Poor and Underserved. 2009; 20:458-72. [PubMed: 19395842]

Colthirst, P.; Momany, E.; Damiano, P.; Warren, J. Utilization of dental services by children in foster care in Iowa. Abstract presented at the 2009 AcademyHealth Annual Research Meeting; Chicago, IL. 2009.

Council on Children With Disabilities, Section on Developmental Behavioral Pediatrics, Bright Futures Steering Committee, Medical Home Initiatives for Children With Special Needs Project Advisory Committee. Identifying infants and young children with developmental disorders in the medical home: an algorithm for developmental surveillance and screening. Pediatrics. 2006; 118:405-420. [PubMed: 16818591]

Cunnion DT, Spiro A 3rd, Jones JA, Rich SE, Papageorgiou CP, Tate A, Casamassimo P, Hayes C, Garcia RI. Pediatric oral health-related quality of life improvement after treatment of early childhood caries: a prospective multisite study. Journal of Dentistry for Children (Chic). 2010; 77:4-11.

Davies GM, Duxbury JT, Boothman NJ, Davies RM, Blinkhorn AS. A staged intervention dental health promotion programme to reduce early childhood caries. Community Dental Health. 2005; 22:118-22. [PubMed: 15984138]

DeVoe JE, Krois L, Stenger R. Do children in rural areas still have different access to health care? Results from a statewide survey of Oregon's food stamp population. Journal of Rural Health. 2009; 25:1-7. [PubMed: 19166555]

Feigal RJ, Jensen ME, Mensing CA. Dental caries potential of liquid medications. Pediatrics. 1981; 68:416-9. [PubMed: 7279470]

Ferguson F, Cinotti D. Home oral health practice: the foundation for desensitizeation and dental care for special needs. Dental Clinics of North America. 2009; 5:375-87. xi. [PubMed: 19269405]

Gift HC, Reisine ST, Larach DC. The social impact of dental problems and visits. American Journal of Public Health. 1992; 82:1663-8. Erratum in: American Journal of Public Health 83 816. [PubMed: 1456343]

Grembowski D, Spiekerman C, Milgrom P. Linking mother and child access to dental care. Pediatrics. 2008; 122:e805-14. [PubMed: 18829778]

Hughes JS, Averill RF, Eisenhandler J, Goldfield NI, Muldoon J, Neff JM, Gay JC. Clinical Risk Groups (CRGs): a classification system for risk-adjusted capitation-based payment and health care management. Medical Care. 2004; 42:81-90. [PubMed: 14713742]

Institute of Medicine. Crossing the Quality Chasm: A New Health System for the 21st Century. 2001.

Keene JJ Jr, Galasko GT, Land MF. Antidepressant use in psychiatry and medicine: importance for dental practice. Journal of the American Dental Association. 2003; 134:71-9. [PubMed: 12555959]

Marinho VC, Higgins JP, Logan S, Sheiham A. Systematic review of controlled trials on the effectiveness of fluoride gels for the prevention of dental caries in children. Journal of Dental Education. 2003; 67:448-58. [PubMed: 12749574]

Marshall TA, Eichenberger-Gilmore JM, Larson MA, Warren JJ, Levy SM. Comparison of the intakes of sugars by young children with and without dental caries experience. Journal of the American Dental Association. 2007; 138:39-46. [PubMed: 17197400] 
Maurer SM, Boggs AM, Mourino AP, Farrington FH. Recall intervals: effect on treatment needs of the handicapped patient: a retrospective study. Journal of Clinical Pediatric Dentistry. 1996; 20:123-6. [PubMed: 8619971]

Mayer ML, Stearns SC, Norton EC, Rozier RG. The effects of Medicaid expansions and reimbursement increases on dentists' participation. Inquiry. 2000; 37:33-44. [PubMed: 10892356]

Nainar SM. Dentists' ranking of Medicaid reimbursement rates as a measure of their pediatric Medicaid participation. ASDC Journal of Dentistry for Children. 2000; 67:422-4. 375-407. [PubMed: 11204067]

Nainar SM, Mohummed S. Diet counseling during the infant oral health visit. Pediatric Dentistry. 2004; 26:459-62. [PubMed: 15460303]

Nunn ME, Braunstein NS, Krall Kaye EA, Dietrich T, Garcia RI, Henshaw MM. Healthy eating index is a predictor of early childhood caries. Journal of Dental Research. 2009; 88:361-6. [PubMed: 19407158]

Patrick DL, Lee RS, Nucci M, Grembowski D, Jolles CZ, Milgrom P. Reducing oral health disparities: a focus on social and cultural determinants. BMC Oral Health. 2006; 15:S4. [PubMed: 16934121]

Pinto-Martin JA, Dunkle M, Earls M, Fliedner D, Landes C. Developmental stages of developmental screening: steps to implementation of a successful program. American Journal of Public Health. 2005; 95:1928-1932. [PubMed: 16195523]

Plutzer K, Spencer AJ. Efficacy of an oral health promotion intervention in the prevention of early childhood caries. Community, Dental and Oral Epidemiology. 2008; 36:335-46.

Vargas CM, Macek MD, Goodman HS, Wagner ML. Dental pain in Maryland school children. Journal of Public Health Dentistry. 2005; 65:3-6. [PubMed: 15751489]

Weintraub JA, Ramos-Gomez F, Jue B, Shain S, Hoover CI, Featherstone JD, Gansky SA. Fluoride varnish efficacy in preventing early childhood caries. Journal of Dental Research. 2006; 85:172-6. [PubMed: 16434737]

Zanin L, Meneghim MC, Assaf AV, Cortellazzi KL, Pereira AC. Evaluation of an educational program for children with high risk of caries. Journal of Clinical Pediatric Dentistry. 2007; 31:246-50. [PubMed: 19161059] 


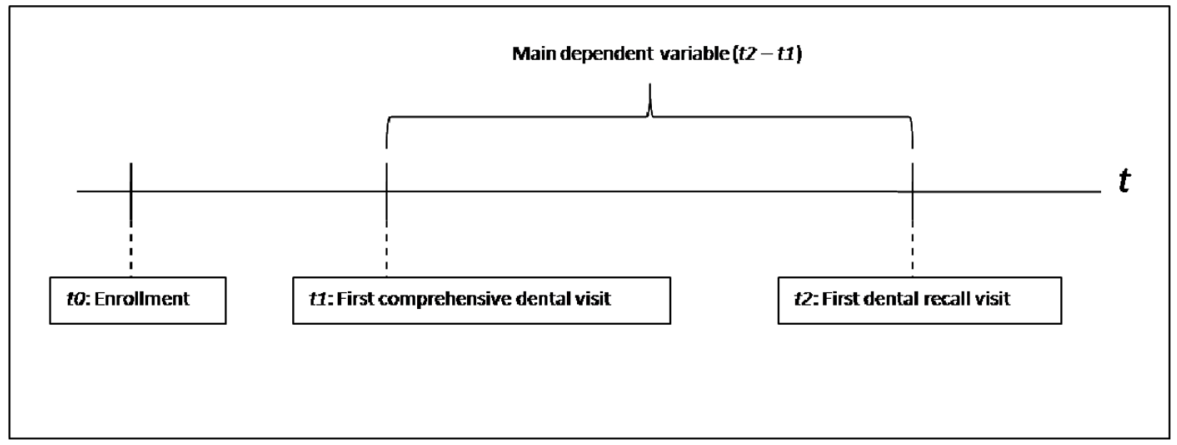

Figure 1.

Description of Dependent Variable (Time to First Dental Recall Visit from the First Comprehensive Dental Visit) 


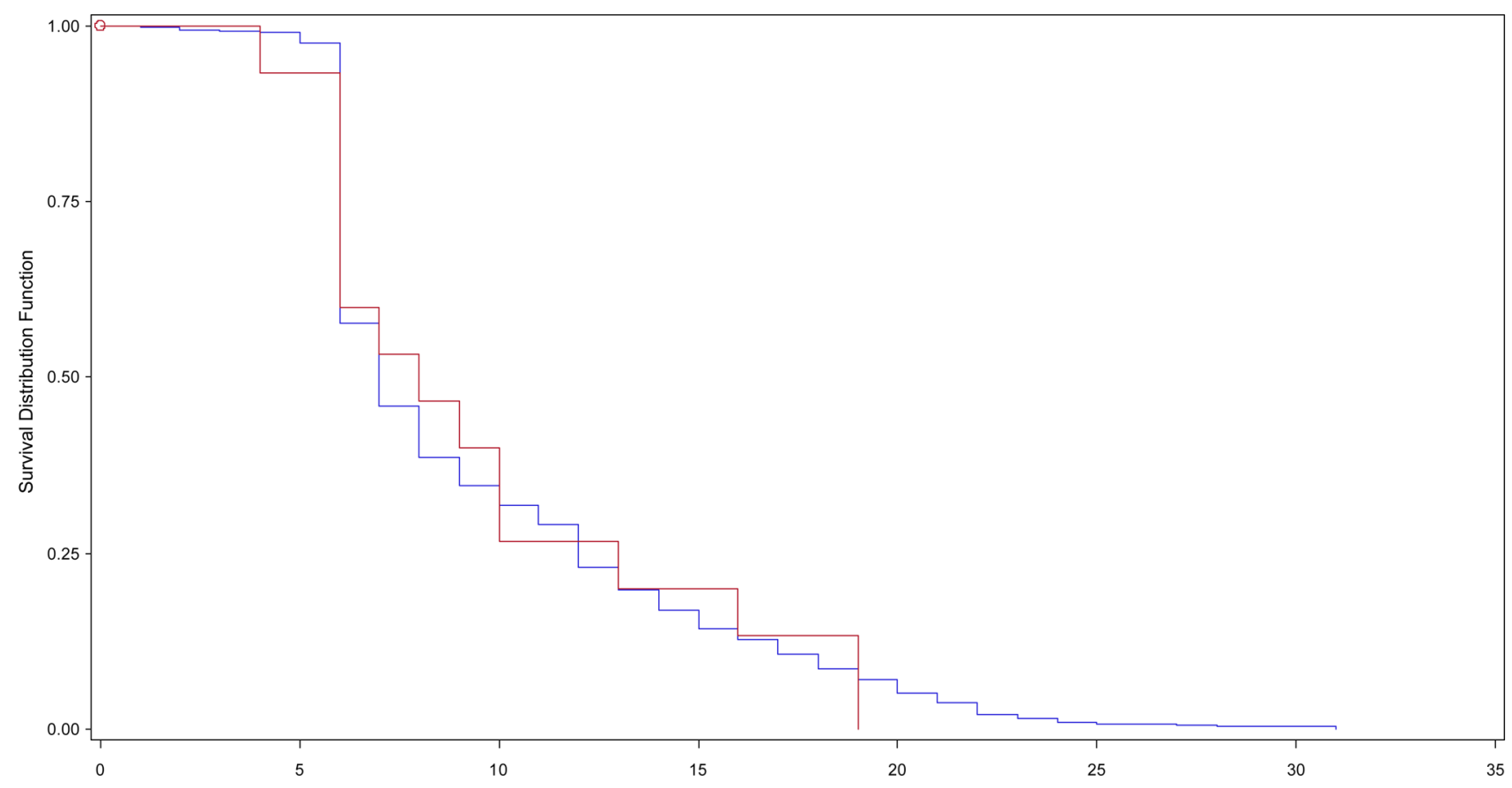

Figure 2.

Kaplan-Meier Survival Curves for Newly-Medicaid Enrolled Children in Iowa Identified with an Intellectual or Developmental Disability (IDD) (red line) and Children without an IDD (blue line) in Calendar Year 2005 and the Time to First Dental Recall Visit in Months 


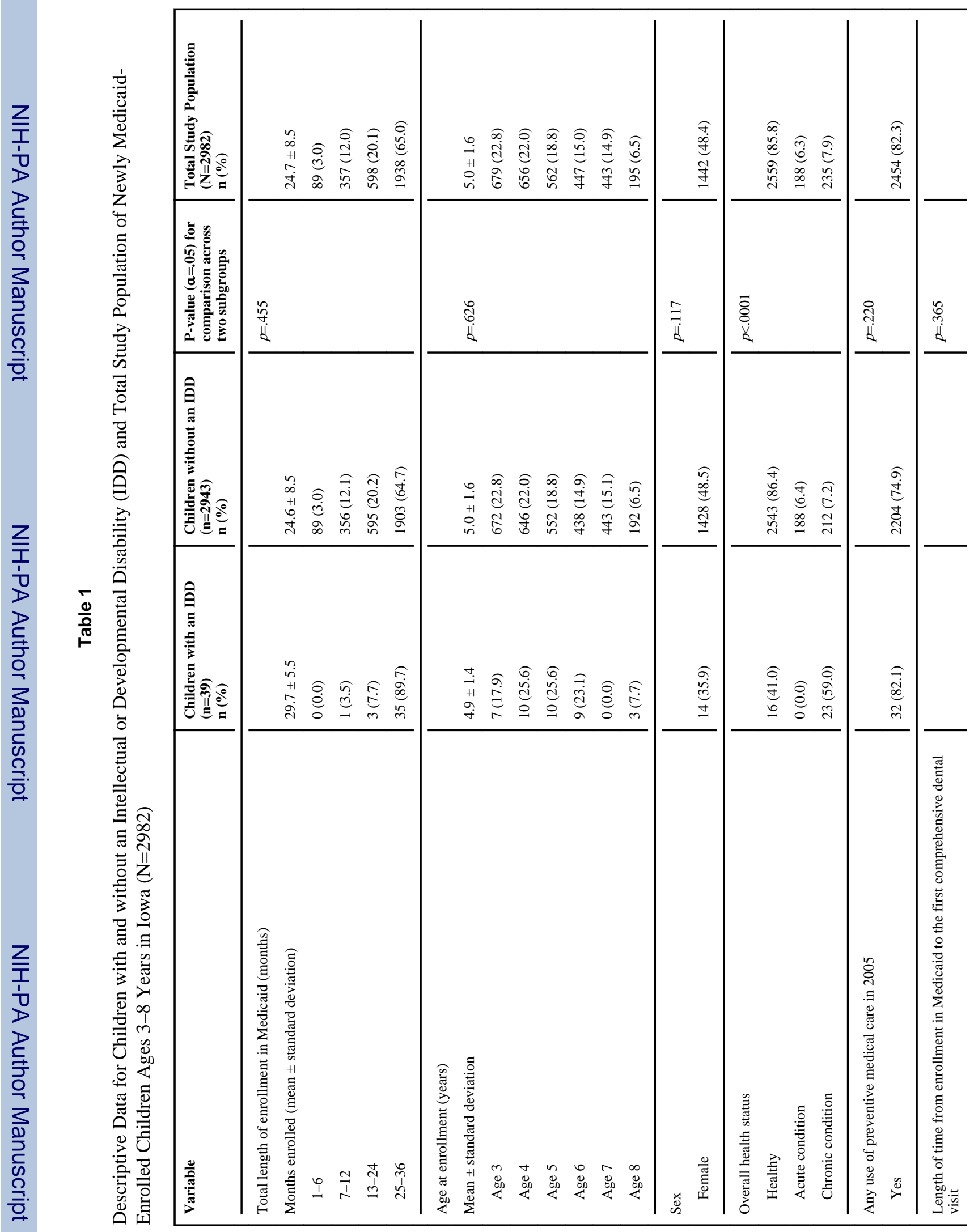




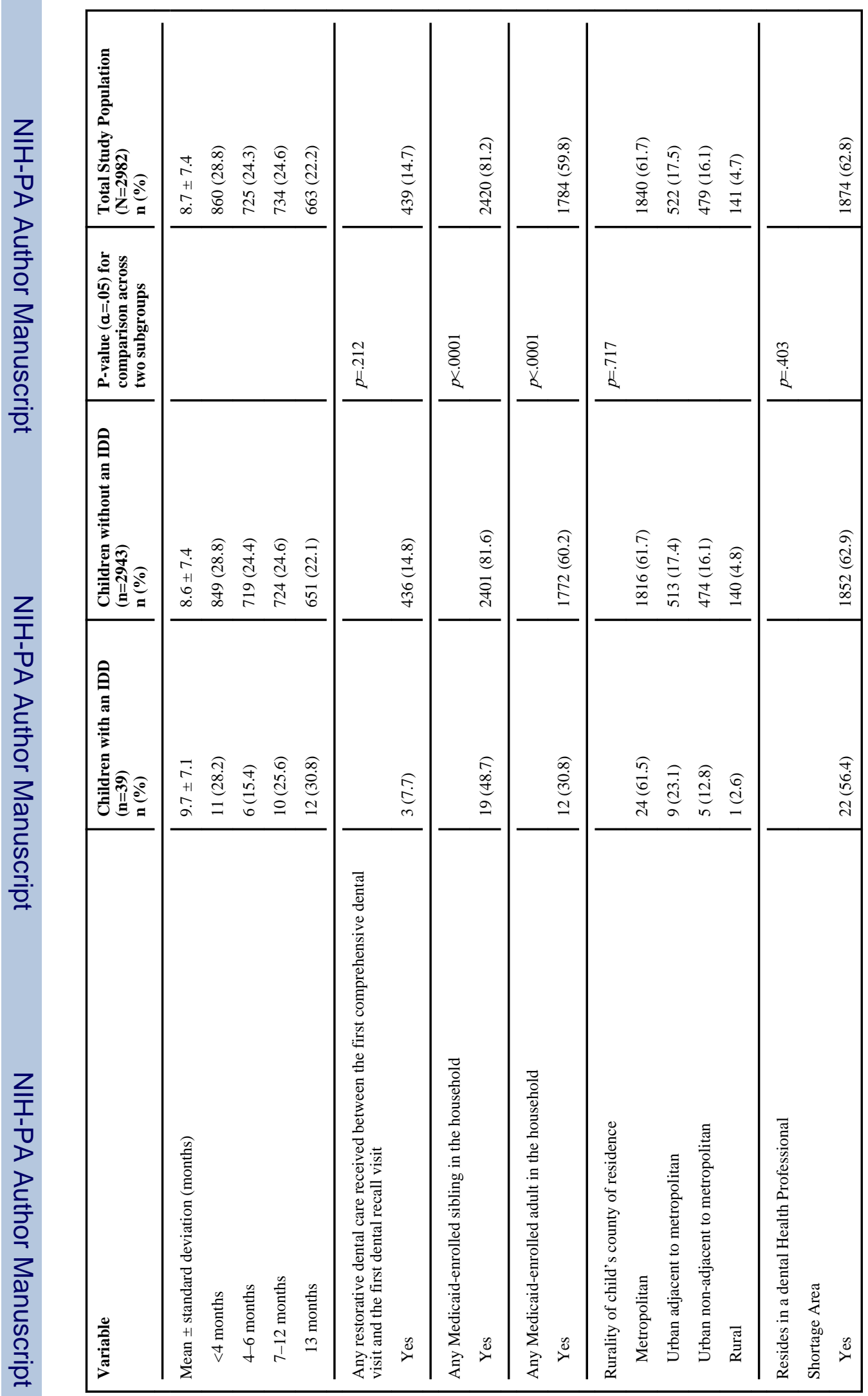

Intellect Dev Disabil. Author manuscript; available in PMC 2013 March 02. 


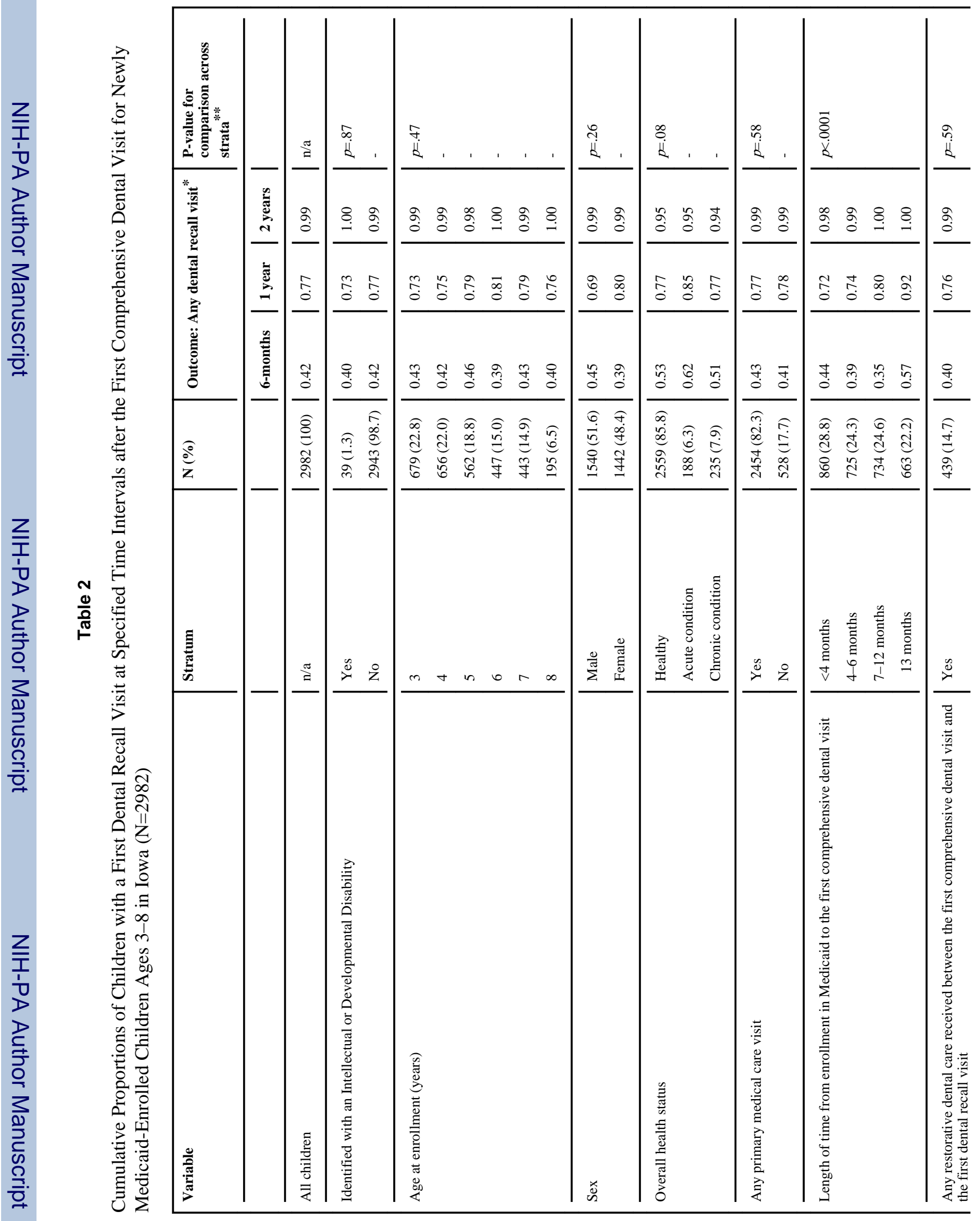




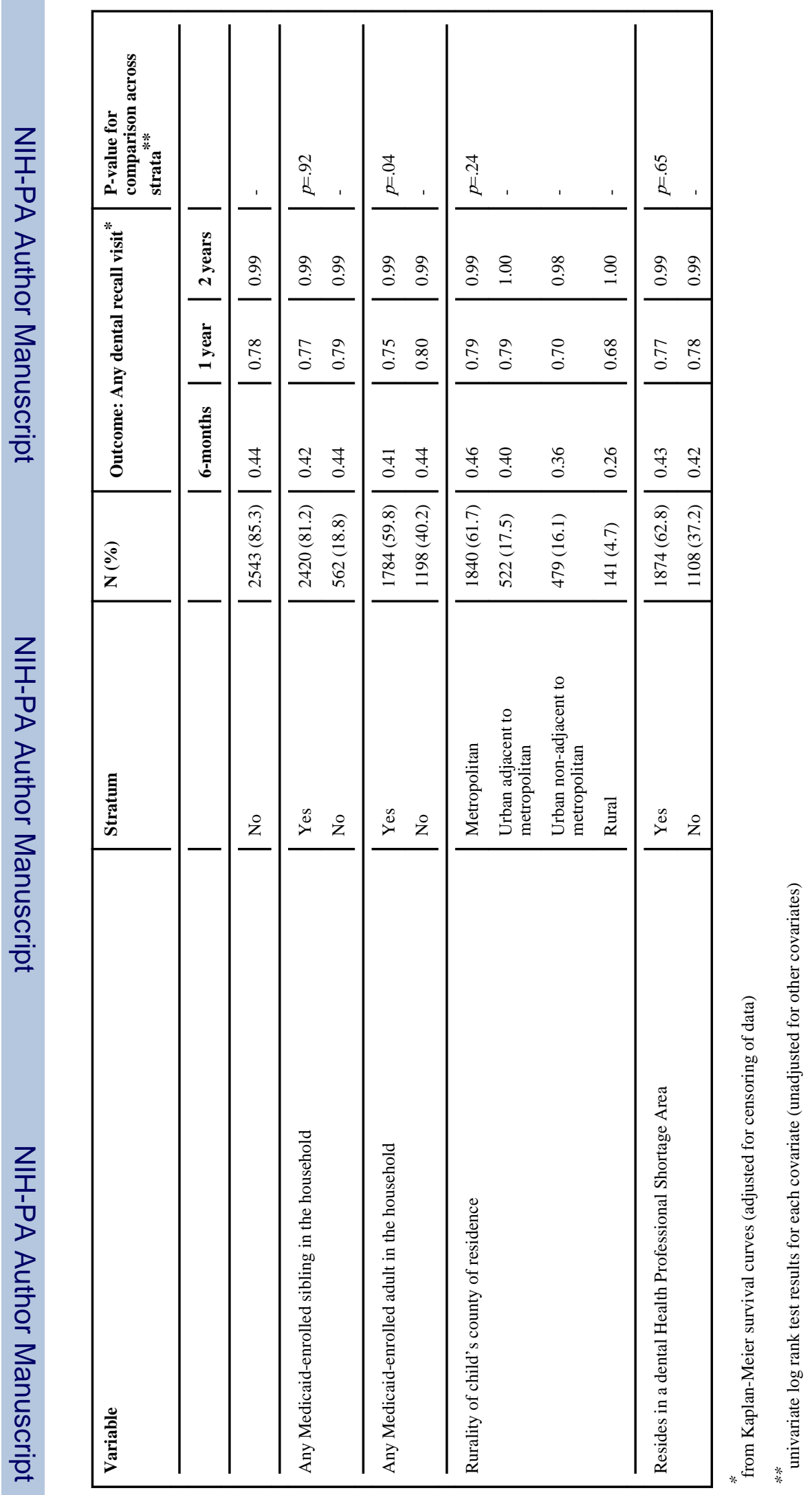

Intellect Dev Disabil. Author manuscript; available in PMC 2013 March 02. 


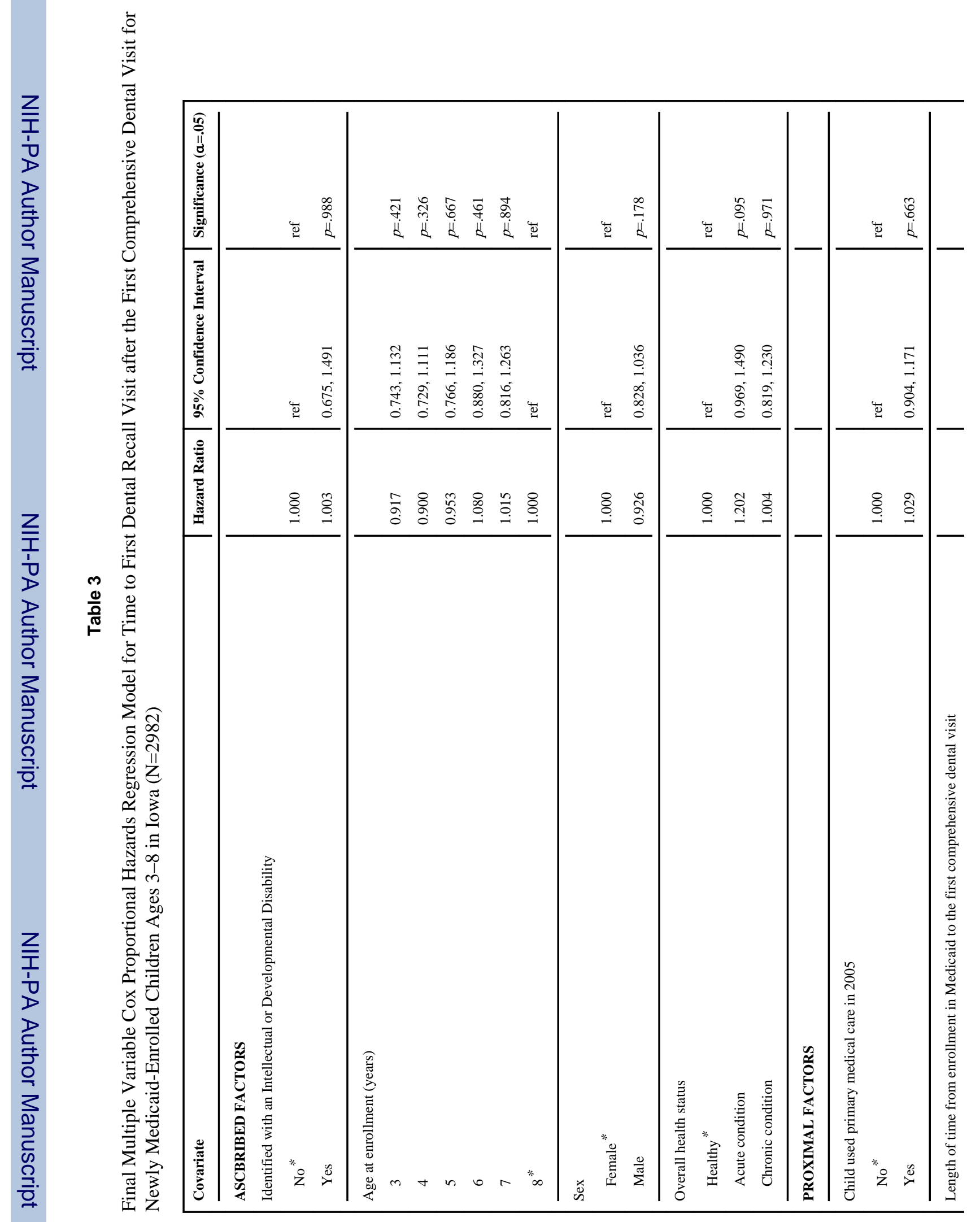

Intellect Dev Disabil. Author manuscript; available in PMC 2013 March 02. 


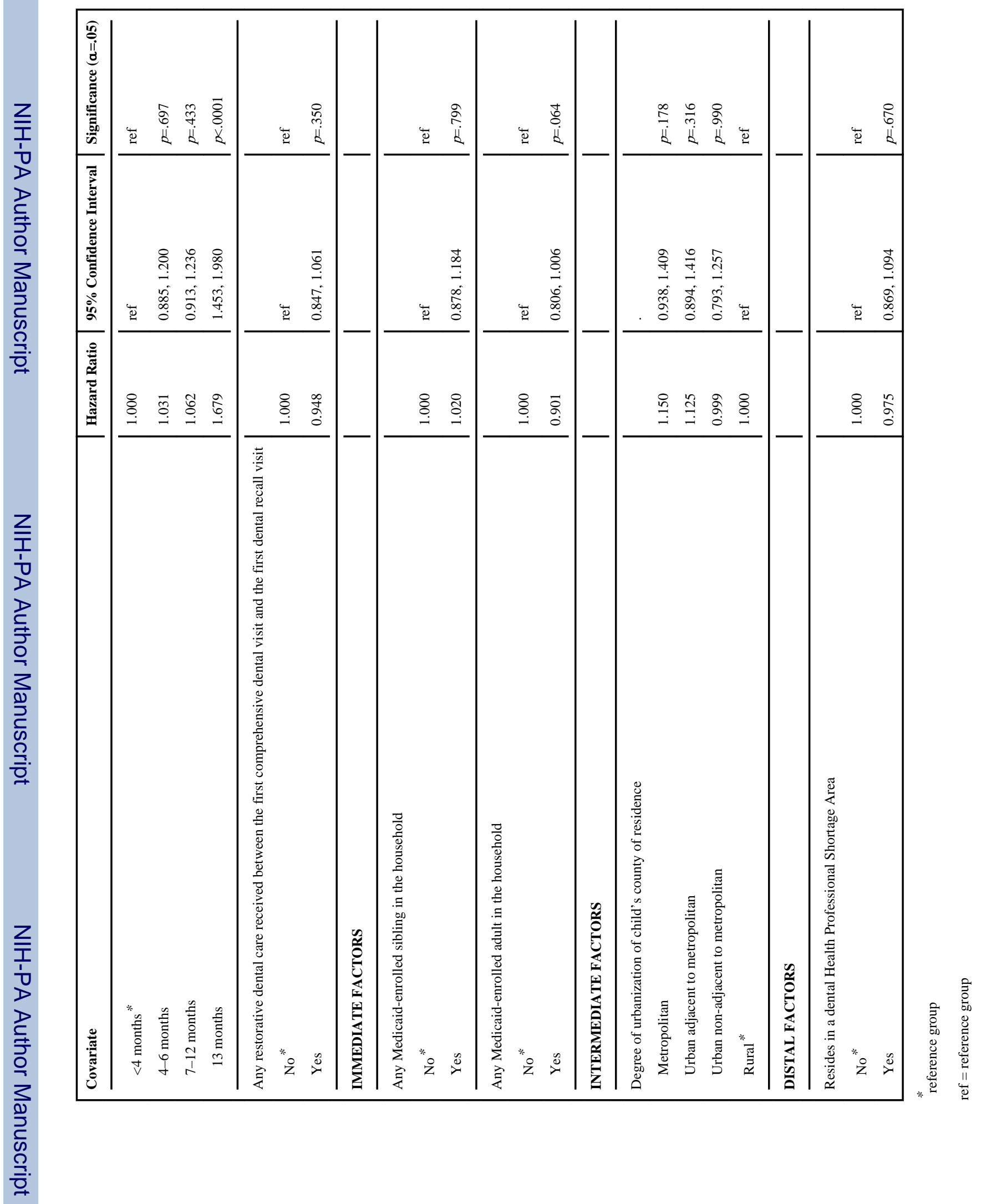

